Cite this: RSC Adv., 2019, 9, 30419

Received 9th April 2019

Accepted 19th September 2019

DOI: $10.1039 / c 9 r a 02645 b$

rsc.li/rsc-advances
Check for updates

\section{Overproduction of lipopeptide biosurfactant by Aneurinibacillus thermoaerophilus HAKO1 in various fed-batch modes under thermophilic conditions $\dagger$}

\author{
Hamidreza Hajfarajollah, ${ }^{a}$ Babak Mokhtarani, (DD *a Azadeh Tohidi, $^{\text {b }}$ \\ Shayesteh Bazsefidpar ${ }^{a}$ and Kambiz Akbari Noghabi (D) *b
}

\begin{abstract}
An efficient lipopeptide biosurfactant (BS) producer, Aneurinibacillus thermoaerophilus HAK01, was isolated from municipal landfill sites. The strain was able to produce about $4.9 \mathrm{~g} \mathrm{~L}^{-1}$ lipopeptide at a thermophilic temperature of $45{ }^{\circ} \mathrm{C}$. After optimization of culture component concentrations using the response surface method, the main focus is to find the most appropriate fed-batch strategy to enhance lipopeptide production by the HAKO1 strain. For this purpose, four fed-batch strategies including (a) $\mathrm{pH}$ stat mode, (b) constant feeding rate strategy, (c) DO-stat mode, and (d) combined feeding strategy were designed. The production of BS was increased systematically from $4.9 \mathrm{~g} \mathrm{~L}^{-1}$ in batch mode to $5.9,7.1$, 8.8 and $11.2 \mathrm{~g} \mathrm{~L}^{-1}$ in each fed-batch mode, respectively. While poor results were obtained in the $\mathrm{pH}$-stat mode, the DO-stat mode showed excellent results in the production of BS. The results of the study confirmed the importance of operational mode, oxygen supply and the kind of feeding strategy in BS production.
\end{abstract}

\section{Introduction}

The rapid development of biotechnology has influenced diverse sectors of the economy. Biosurfactants (BS) as one of the important bio-products have many applications in the environmental, food, agriculture, petroleum, paper/pulp and pharmaceutical industries. These amphiphilic compounds, which are produced by microbial cells, ${ }^{1}$ have several benefits compared to synthetic surfactants, including low toxicity, high biodegradability, better environmental compatibility, specific activity at extreme temperatures, $\mathrm{pH}$ and salinity, and the ability to be synthesized from renewable feed stocks. ${ }^{2,3}$

In order to apply current biotechnology research to industry, new processes must be developed to transform current research into viable market products. ${ }^{4}$ Specifically, attention must be directed toward the industrial processes of cultivation of microorganisms. Fed-batch cultivation has been widely employed for further productivity improvement of various bioproducts including primary and secondary metabolites, proteins and biopolymers. ${ }^{5}$ Fed-batch fermentation is used to

${ }^{a}$ Chemistry and Chemical Engineering Research Center of Iran, P. O. Box 14335-186, Tehran, Iran. E-mail: mokhtaranib@ccerci.ac.ir; Fax: +98 2144787780; Tel: +98 2144787770

${ }^{b}$ National Institute of Genetic Engineering and Biotechnology, P. O. Box 14155-6343, Tehran, Iran.E-mail: Akbari@nigeb.ir

$\dagger$ Electronic supplementary information (ESI) available. See DOI:

prevent or reduce substrate-associated growth inhibition by controlling nutrient supply. ${ }^{5,6}$ During fed-batch cultivation, one or more nutrients are supplied to the fermenter while cells and products remain in the fermenter until the end of the operation.

When designing a fed-batch fermentation, the most intriguing variable which needs to be defined is the feeding profile. The control-system development for feeding strategy is not straightforward due to: (1) the lack of accurate models describing cell growth and product formation; (2) the nonlinear nature of the bioprocess; (3) the slow process response, and (4) deficiency of reliable on-line sensors. ${ }^{7}$ Fortunately, there are various useful methods, which have been described to determine and optimize the feeding profile. In general, the feeding methods may be classified into two main types: those without any kind of feedback control and those relying on some form of a feedback control mechanism. The latter type of feeding methods uses an online measured fermentation variable to control the feeding. Constant rate feeding, an increasing rate feeding, and exponential feeding are among the fed-batch processes without feedback control, while DO-stat and pH-stat modes are among the fedbatch processes with feedback control. ${ }^{8}$

In literature, there are very few works concerning the production of BSs in fed-batch mode. Zhu et al. (2012) studied a pH-stat process for the production of rhamnolipid biosurfactant by $P$. aeruginosa. A yield of $24.06 \mathrm{~g} \mathrm{~L}^{-1}$ in batch fermentation increased to $28.8 \mathrm{~g} \mathrm{~L} \mathrm{~L}^{-1}$ in $\mathrm{pH}$-stat fed-batch fermentation, which showed $19.7 \%$ improvement. ${ }^{9}$ In another 
work, Guez et al. studied the production of lipopeptide biosurfactant "mycosubtilin" from Bacillus subtilis with overflowing exponential fed-batch cultures. ${ }^{10}$ The feeding strategy was developed to keep the specific growth rate at constant value. This kind of strategy was led to the production of $1.27 \mathrm{mg}$ of mycosubtilin per $\mathrm{g}$ of dried biomass per $\mathrm{h}$. In addition in our previous work, ${ }^{11}$ the production of rhamnolipid under tight DO control in a fedbatch mode was evaluated in which 4.8-fold improvement was achieved compared to the batch experiments. However, there is not any single report to compare different fed-batch strategies for BS production in detail.

In the present study, the main goal is to find a fed-batch strategy to increase the BS production by Aneurinibacillus thermoaerophilus HAK01. This strain has been isolated and identified from urban waste during a comprehensive study. The BS production improved step by step in different feeding strategies including; pH-stat operation, DO-stat operation, constant rate feeding and combined feeding.

\section{Experimental}

\subsection{Sampling and isolation of BS producer bacteria}

Samples were collected from urban wastes of the Kahrizak site in the south of Tehran. The samples were inoculated in nutrient broth medium and incubated on a rotary shaker (Kuhner, Germany) at $180 \mathrm{rpm}$ for 2 days and in two different temperatures 30 and $45{ }^{\circ} \mathrm{C}$. Serial dilutions of cultures were done followed by spreading on nutrient agar plates. Single colonies were obtained and isolated in another nutrient agar plates. The ability of purified colonies to produce BS were examined by oil spreading test and measurement of medium surface tension. For this purpose, the purified colonies were cultured in two different media. The composition of these two media were ( $\mathrm{g}$ $\mathrm{L}^{-1}$ ): (1) $\mathrm{NaNO}_{3} 3, \mathrm{MgSO}_{4} \cdot 7 \mathrm{H}_{2} \mathrm{O} 0.25, \mathrm{KH}_{2} \mathrm{PO}_{4} 0.25$, yeast extract 1, and sunflower oil 20, final pH: 6.9. (2) $\mathrm{NH}_{4} \mathrm{NO}_{3} 4.5, \mathrm{MgSO}_{4}$ $\cdot 7 \mathrm{H}_{2} \mathrm{O} 0.2, \mathrm{KH}_{2} \mathrm{PO}_{4} 0.25, \mathrm{Na}_{2} \mathrm{HPO}_{4} 5.6, \mathrm{Fe}_{2} \mathrm{SO}_{4} \cdot 7 \mathrm{H}_{2} \mathrm{O} 0.083$, and glucose 20 , final pH: 7.05 .

\subsection{Molecular identification of isolated strains}

Seven BS producers were selected and identified using 16S rRNA gene sequence analysis for molecular identification. DNA extraction was performed according to the previous work. ${ }^{\mathbf{1 2}}$ Three sets of primers: 27f (5'-AGAGTTTGATCCTGGCTCAG), 1492r (5'-TACGGTTACCTTGTTACGAC TT) and V3 and V6 primers were used to amplify the V3 and V6. ${ }^{13}$ The PCR amplification conditions were: initial denaturation at $95{ }^{\circ} \mathrm{C}$ for $5 \mathrm{~min}$, followed by 25 cycles each of $94{ }^{\circ} \mathrm{C}$ for $1 \mathrm{~min}, 55^{\circ} \mathrm{C}$ of annealing for $45 \mathrm{~s}$, and a $45 \mathrm{~s}$ extension at $72{ }^{\circ} \mathrm{C}$. The PCR products were sequenced on an ABI Prism 377 automatic sequencer (Applied Biosystems, CA, USA). Sequence homologies were examined using BLAST version 2.2.12 of the National Center for Biotechnology Information. ${ }^{\mathbf{1 4}}$

\subsection{Extraction of biosurfactant}

The produced biosurfactant was extracted from the culture broth with the method of acid precipitation followed by solvent extraction reported elsewhere. ${ }^{12}$ Briefly, the culture broth was centrifuged $\left(6000 \times g, 10 \mathrm{~min}, 4{ }^{\circ} \mathrm{C}\right)$ to remove the cells. Then, $\mathrm{pH}$ was adjusted to 2 by $3 \mathrm{M} \mathrm{HCl}$ in order to precipitate the biosurfactant. After keeping it overnight, the crude biosurfactant was obtained by centrifugation $(18000 \times g, 30 \mathrm{~min}, 4$ $\left.{ }^{\circ} \mathrm{C}\right)$. The crude biosurfactant was purified using several times extraction by ethyl acetate.

\subsection{Kinetics of the growth and BS production in batch mode}

In a batch operation, the kinetics of the cell growth and lipopeptide production of the selected strain were obtained in the medium containing ( $\mathrm{g} \mathrm{L}{ }^{-1}$ ): $\mathrm{NaNO}_{3} 3, \mathrm{MgSO}_{4} \cdot 7 \mathrm{H}_{2} \mathrm{O} \quad 0.25$, $\mathrm{KH}_{2} \mathrm{PO}_{4} \quad 0.25$, yeast extract 1 , and sunflower oil 20. The concentration of biomass, lipopeptide production, surface tension of supernatant and the results of the oil spreading test were measured via periodic sampling. This experiments were performed in $250 \mathrm{~mL}$ flasks containing $100 \mathrm{~mL}$ of the production medium at $180 \mathrm{rpm}$ and $45^{\circ} \mathrm{C}$ for $144 \mathrm{~h}$. Experiments were repeated for three times and the averages were reported.

\subsection{Optimization of culture medium}

Central composite design (CCD) was applied to study the effect and interactions of the various essential nutrients namely sunflower oil, $\mathrm{NaNO}_{3}, \mathrm{MgSO}_{4} \cdot 7 \mathrm{H}_{2} \mathrm{O}$, and yeast extract in five levels. The range and the levels of the factors are specified in Table 1 . This procedure was carried out to optimize the medium components in order to achieve the maximum lipopeptide production by A. thermoaerophilus. Minitab software version 16, was used for experimental design and statistical analysis of the data. A total of 31 experiments were employed with 2 replicates at the center points. The statistical combinations of the critical media components are detailed in (Table S1 $\dagger$ ).

\subsection{Cultivation of HAKO1 in bioreactor}

Fed-batch fermentation could be an effective way for enhancing production using low solubility substrate such as lipids. Considering the fact that most vegetable oils and lipids (as carbon source) are practically insoluble in water, it is expected that the biosurfactant productivity could be enhanced by fedbatch fermentation. ${ }^{15}$ An attempt has been made to develop an efficient fed-batch strategy to enhance the lipopeptide production. In order to achieve this goal, four feeding strategies have been evaluated: (1) pH-stat (2) DO-stat, (3) constant rate feeding and (4) combined feeding. The initial culture composition will be as for the optimized batch operation. Cultivations

Table 1 Experimental ranges and variables for optimization by RSM

\begin{tabular}{llllllll}
\hline & & \multicolumn{2}{l}{ Levels } \\
\cline { 3 - 7 } Symbol & Factors & $-\alpha$ & -1 & 0 & 1 & $+\alpha$ \\
\hline A & Sun flower oil $\left(\mathrm{g} \mathrm{L}^{-1}\right)$ & 8.5 & 10 & 15 & 20 & 21.5 \\
B & Magnesium sulfate $\left(\mathrm{g} \mathrm{L}^{-1}\right)$ & 0.11 & 0.2 & 0.5 & 0.8 & 0.89 \\
C & Sodium nitrate $\left(\mathrm{g} \mathrm{L}^{-1}\right)$ & 1.7 & 2 & 3 & 4 & 4.3 \\
D & Yeast extract $\left(\mathrm{g} \mathrm{L}^{-1}\right)$ & 0.27 & 0.5 & 1.25 & 2 & 2.22
\end{tabular}


were performed in a $5 \mathrm{~L}$ fermenter (Minifors, Switzerland) with working volume of $2 \mathrm{~L}$ at $45{ }^{\circ} \mathrm{C}$, and $\mathrm{pH} 7$. In all experiments, culture was inoculated with $3 \% \mathrm{v} / \mathrm{v}$ of the seed. The aeration rate of 1-1.5 vvm and the agitation speed of 200-1200 rpm was used to set the dissolved oxygen (DO) concentration at a specified value. The DO concentration was controlled on about $25 \%$ by changing agitation speed and aeration rate. The $\mathrm{pH}$ of culture medium was adjusted using $4 \mathrm{M} \mathrm{NaOH}$ and $20 \%$ phosphoric acid. DO, pH, and temperature were monitored using probes mounted on the fermenter. Silicon oil was used as antifoam during lipopeptide fermentation in the fed-batch process. All experiments were done during 4-5 days. During the experiments, samples were taken from the fermentation broth and analyzed to determine the biomass growth, substrate consumption, and lipopeptide production.

2.6.1. Preliminary experiments. In order to find the behavior of cell growth, BS production and oxygen consumption in the fermenter as well as to determine the effect of culture components on cell growth and lipopeptide production, some preliminary experiments were performed in the fermenter. After these experiments, four different fed-batch strategies were designed as follows:

2.6.2. $\quad$ pH-stat strategy. In $\mathrm{pH}$-stat feeding, the automatic $\mathrm{pH}$ control system of the batch operation was switched off. ${ }^{16}$ The limiting substrate was fed when the $\mathrm{pH}$ increased above 7.3 indicating a depletion of the substrate. For each feeding, an appropriate amount of culture medium was pumped out of the fermenter to keep the final volume of the broth in constant level. It should be mentioned that $\mathrm{NaOH} 4 \mathrm{M}$ and phosphoric acid $20 \%$ were used to control the $\mathrm{pH}$ of the medium in all experiments.

2.6.3. Constant feeding-rate. Constant rate feeding is relatively easy to implement and has been widely used in producing biomaterials. Two kinds of constant rate feeding strategies were conducted based on the preliminary experiments. At the first experiment, the oil concentration was maintained above $15 \mathrm{~g}$ $\mathrm{L}^{-1}$ during the growth phase using constant feeding of oil into the fermenter. In the second experiment, besides constant feeding of oil, constant rate feeding of yeast extract was also applied. In this step the aim is to increase cell dry weight (CDW). During the experiments, samples were withdrawn from the fermentation broth at appropriate time intervals and analyzed to determine the bacterial growth, substrate consumption and lipopeptide production.

2.6.4. DO-stat strategy. Most of the modern fermentation systems have the DO controlled substrate feed program built in the control unit. Therefore, DO-stat could be a great choice for initial substrate limited fed-batch fermentation for production of biomaterials. The commonly used DO-stat control feeding strategy for fed-batch fermentations involves adding the substrate when the dissolved oxygen (DO) level rises above the set-point value due to depletion of the substrate. In the present experiments, the set-point value for DO was considered as $25 \%$. At the time of DO reduction below the set point, it is adjusted by changing the agitation speed or aeration rate.

2.6.5. Combined feeding. Feeding strategies vary in complexity, reliability and the expense of implementation. Constant rate feeding, increasing rate feeding and feeding methods based on measurement of dissolved oxygen concentration are among the easiest to implement and are reliable. However, the feeding strategy may be modified during the course of a fed-batch fermentation to achieve better outcomes. Therefore, in this part of experiments, a combined feeding strategy was designed to improve bacterial growth and BS productivity. In the combined feeding strategy, DO-stat mode was used as a base, and the feeding of sunflower oil, yeast extract and $\mathrm{MgSO}_{4}$ from concentrated tanks was performed.

\subsection{Analysis and measurements}

Cellular growth is expressed in terms of optical density or dry cell weight (DCW). These two parameters can be converted to each other by the equation of a calibration curve constructed between them: DCW $\left(\mathrm{g} \mathrm{L}^{-1}\right)=0.36 \times \mathrm{OD}_{600}$. The optical density was measured at $600 \mathrm{~nm}$ by UV-visible spectrophotometer (PerkinElmer, model Lambda 25, USA) during different time intervals.

In order to measure the oil consumption, $10 \mathrm{~mL}$ sample was retrieved from fermenter during specific time intervals and the residual oil was extracted using $n$-hexane. The top layer phase, containing sunflower oil was separated by separation funnel, evaporated with a rotary vacuum evaporator (Thermo Electron Corporation, Heraeus Vacutherm), and weighed.

After removing the residual oil by $n$-hexane extraction, the produced BS was extracted from culture medium with the method of acid precipitation followed by solvent extraction reported previously. ${ }^{17}$

Surface tension (ST) was measured using Krüss K100MK2 tensiometer (Krüss GmbH, Germany) using Wilhelm plate technique. The tensiometer was calibrated against double distilled water. The standard procedure of oil spreading test (OST) was performed according to our previous work to find lipopeptide activity. ${ }^{17}$ OST is also sometimes used as a reliable way to measure the concentration of BSs.

\section{Results and discussion}

\subsection{Isolation and identification of BS producer strains}

140 different colonies were obtained after purification of bacteria from the collected samples of urban waste. All of these colonies were cultured in two different media as described in Section 2.1. Oil spreading test (OST) was done for three days and surface tension of the media was measured in the 3rd day as an indicator for the presence of BS in the culture. The results of OST can be seen in Table S2.† Among 140 purified colonies, seven bacteria (the bacterial numbers: 31 , 43, 47, 81, 91, 96, 127) with the best surface activity in their culture were selected Table S2. $\dagger$ All these bacteria were identified by PCR method followed by gene sequencing according to Section 2.2. These bacteria were submitted in and accepted by National Center for Biotechnology Information (NCBI) respectively as (1) P. aeruginosa HAK01, accession no. KP100523, (2) P. aeruginosa HAK02, accession no. KR025471, (3) P. aeruginosa HAK03, accession no. KR025472, (4) Aneurinibacillus thermoaerophilus HAK01, accession no. KR025473, (5) Enterococcus gallinarum HAK 01, 
accession no. KP100524, (6) Serratia marcescens HAK 01, accession no. KR025474 and (7) Bacillus tequilensis HK 01, accession no. KP144873.

Among these bacteria, Aneurinibacillus thermoaerophilus HAK01 was selected for further studies. It should be mentioned that the physiochemical characterization of the BS from the same strain has been performed in our previous work ${ }^{9}$ and the produced BS characterized as lipopeptide. Therefore, the aim of this work is mainly the improvement of the lipopeptide production by the selected strain.

All the above-mentioned strains were isolated in $30^{\circ} \mathrm{C}$ except A. thermoaerophilus which was isolated in $45^{\circ} \mathrm{C}$. Therefore, this strain is considered a thermophilic bacterium.

\subsection{Kinetic studies in batch mode}

The specific bioprocess parameters for producing lipopeptide biosurfactant using $A$. thermoaerophilus in batch mode is presented in Fig. 1. As can be observed, the cell growth initiated just after inoculation and reached to the optical density of 3 after two days. The lipopeptide production was increased during cultivation time and reached its maximum level of about $4.9 \mathrm{~g} \mathrm{~L}^{-1}$ after 96 hours. As lipopeptide started to increase, the surface tension (ST) of the medium reduced from 72 to about $40 \mathrm{mN} \mathrm{m}^{-1}$ after $30 \mathrm{~h}$. ST remained at a constant value of $40 \mathrm{mN} \mathrm{m}^{-1}$ for 5 days. In the cultivation of some strains, ST will be increased after a period of time as observed for the BS produced by $P$. freudenreichii. ${ }^{17}$ In such cases, the BS may be consumed by the strain itself.

The diameter of clear zone in the oil spreading test (OST) is an indicator of the surface activity of the BS. ${ }^{17}$ It can be also related to the concentration of produced BS. As can be seen in Fig. 1, this parameter increases with increase in BS production and reached its maximum level after four days.

\subsection{Optimization of culture condition}

The CCD design along with the corresponding experimental values of the lipopeptide production is given in Table S1. $\dagger$
Analysis of variance (ANOVA) of the model, the corresponding $p$ values, along with the parameter estimate for the lipopeptide production is shown in Table S3. $\dagger$ ANOVA results indicated that the model was highly significant, as the $\mathrm{F}$ value for the model was $17.58(p<0.0001)$. For a good statistical model, the $R^{2}$ value should also be close to 1.0 where a value of 0.7 indicates the weakness of the model. ${ }^{18}$ The value of the correlation coefficient, $R^{2}(93.89 \%)$ showed that the regression model provides an accurate description of the experimental data. A reasonable agreement between predicted $(85.40 \%)$ and adjusted $(88.55 \%)$ was also observed. These evaluations confirmed that the model could be used for the prediction of lipopeptide production within the given range of variables.

The parameter estimate and the corresponding $p$-values suggest that, among the test variables, all the factors are significant and have an important effect on lipopeptide production Table $\mathrm{S} 3 \uparrow$ except for $\mathrm{NaNO}_{3}$. The corresponding $p$ value for $\mathrm{NaNO}_{3}$ is about 0.4. It shows that $\mathrm{NaNO}_{3}$ has the lowest influence on lipopeptide production. Among mutual interaction between variables, almost all interactions are significant $(p$-value $<0.05)$ except for oil and yeast extract $(p$ value $=0.1$ ). The response surfaces for interaction between components are illustrated in Fig. S1. $\dagger$ Using response optimizer option of Minitab software and analyzing response surface plots, the optimum levels for sunflower oil, sodium nitrate, magnesium sulfate, and yeast extract were determined to be $18.5,2.4$, and 0.92 and $1.26 \mathrm{~g} \mathrm{~L}^{-1}$, respectively. Using these optimized values, the optimization resulted in $6.6 \mathrm{~g} \mathrm{~L}^{-1}$ lipopeptide production i.e. $34 \%$ improvement compared to the non-optimized batch condition.

\subsection{Fed-batch studies}

3.4.1. Preliminary experiments. In the preliminary experiments, the aim is to get an overview on the behavior of cell growth and variation of parameters over time in the fermenter. Fig. 2A shows the changes in Dissolved Oxygen (DO)

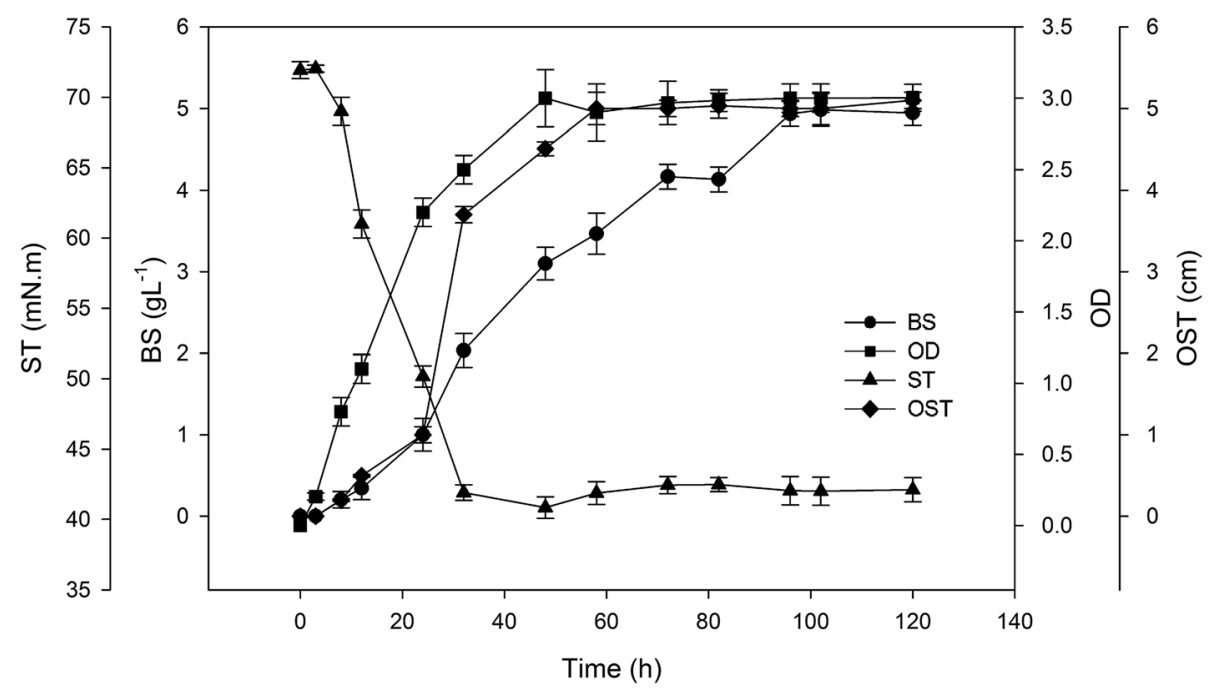

Fig. 1 Kinetics of the growth and BS production in batch mode by A. thermoaerophilus HAKO1. 
concentration, Optical Density (OD), and lipopeptide production in the fermenter in the first ten hours of cultivation. As can be seen in this figure, after a very short lag period, the DO concentration starts to decrease. This represents that the cell growth in the fermenter has been initiated. However, after 110 minutes, the DO concentration increased suddenly with intense steep and reached the maximum level. DO remains at the maximum level for another 240 minutes and decreased again. This observation showed that at the beginning of inoculation, the cell growth is started due to the presence of culture components. After about 2 hours, one of the medium components might have been depleted, so the DO concentration starts to increase. In order to find the consumed component, another experiment was performed. In this experiment, by increasing
DO, culture components were pumped into the fermenter from concentrated feed tanks. Fig. 2B shows that concentrated aqueous solutions of $\mathrm{NaNO}_{3}, \mathrm{MgSO}_{4}$, and $\mathrm{KH}_{2} \mathrm{PO}_{4}$ did not lead to a reduction of DO concentration. However, just after pumping the concentrated aqueous solution of yeast extract to the fermenter, DO concentration was dropped and the strain started to grow. This observation shows that the cell growth at the beginning of inoculation is due to the presence of yeast extract. After consumption of yeast extract, the cell growth stopped until the strain adapted to sunflower oil. However, it should be noted that there might be a complicated relationship between oil and one of the components in yeast extract that can affect the cell growth. However, the results show that yeast extract is one of the main components of culture medium.
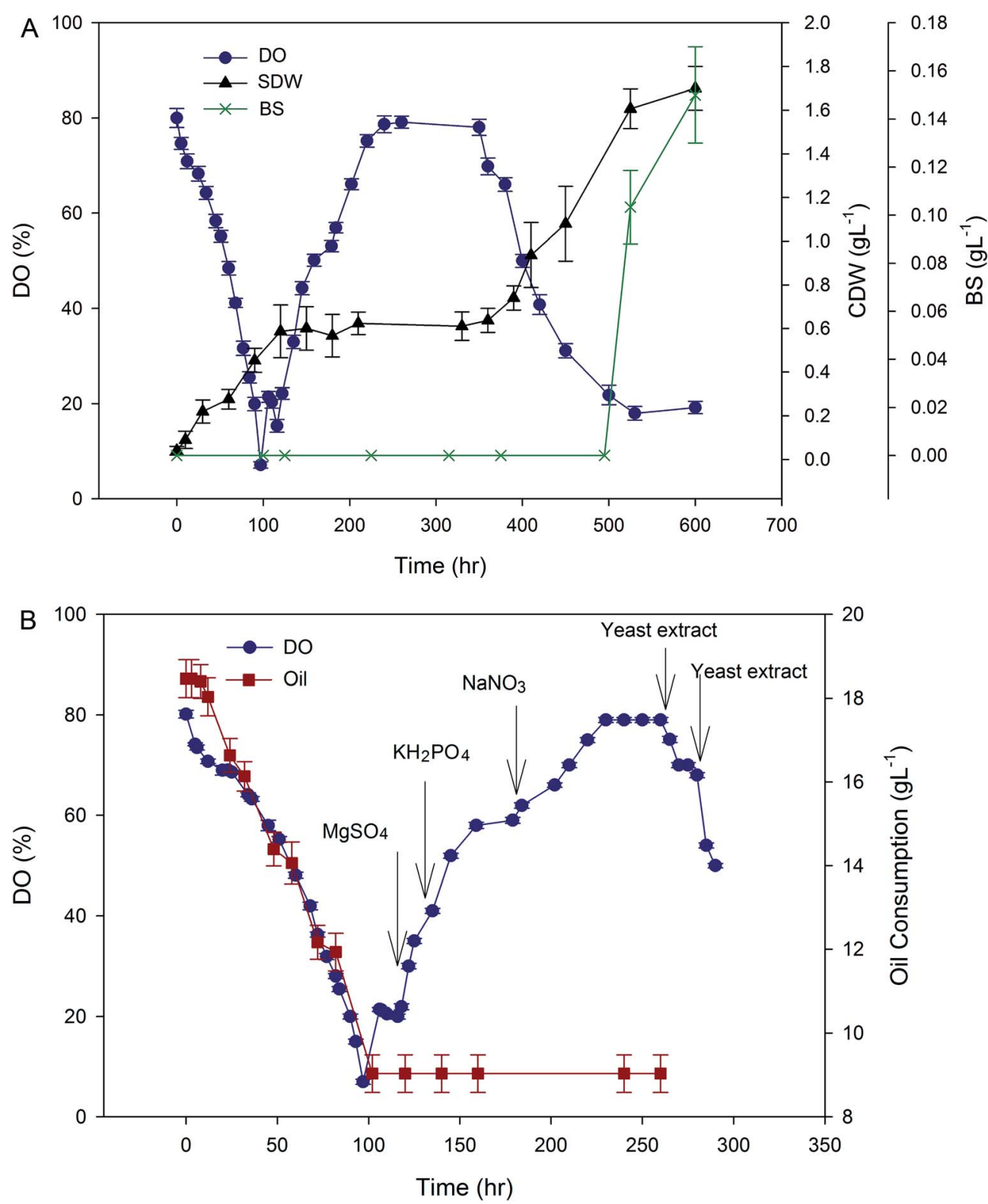

Fig. 2 (A) Primary evaluation of changes in DO concentration, OD, and lipopeptide production in the fermenter, when A. thermoaerophilus grown on sunflower oil as a sole carbon source, (B) effect of feeding of different media component on dissolved oxygen and oil consumption during first hours of the cultivation. 
In order to find the importance of each media component on cell growth and lipopeptide production, a complementary experiment was designed. In these experiments, one of the medium components was removed from culture formulation. The results are presented in Table 2 . As can be seen, without the presence of $\mathrm{MgSO}_{4}$ in the culture medium, the significant cell growth and lipopeptide production were not obtained. Similar results were obtained in absence of sunflower oil as a carbon source in the culture medium. This shows that $\mathrm{MgSO}_{4}$ and oil are essential for cell growth and lipopeptide production. Lack of yeast extract in the culture medium reduced the cell growth and lipopeptide production. In this case, the lipopeptide production was $3.4 \mathrm{~g} \mathrm{~L}^{-1}$ and up to 6 hours the cell growth was not observed. After adaption of the HAK01 strain with a carbon source, cell growth was started. Lack of $\mathrm{NaNO}_{3}$ or $\mathrm{KH}_{2} \mathrm{PO}_{4}$ in the medium only slowed down the cell growth and reduced the lipopeptide production a little. The results of these primary experiments will be used in further main fed-batch experiments.

3.4.2. $\mathbf{p H}$-stat. The result of the fed-batch operation in the $\mathrm{pH}$-stat mode of feeding has been shown in Fig. S2. $\dagger$ The increase in $\mathrm{pH}$ corresponds to the depletion of carbon source or other main components. Another reason can be related to extracellular materials released by bacteria. For example, Bacillus strains can easily decompose proteins that can lead to the release of ammonium. Therefore, the $\mathrm{pH}$ change can also be due to switch of culture's metabolism to protein decomposition. ${ }^{\mathbf{1 9}}$

As indicated in Fig. S2, $\dagger$ except for the initial hours of the cultivation, in which $\mathrm{pH}$ was adjusted by yeast extract feeding, no variation in $\mathrm{pH}$ was observed after about 6 hours. In fact, after the primary feedings demarcated by the arrows in Fig. S2, $\dagger$ the expected increase in $\mathrm{pH}$ was not observed to guide the next feeding. Therefore, feeding cannot be performed precisely. This is because of the medium dependent characteristic of $\mathrm{pH}$-stat strategy. ${ }^{19}$ In fact, the pH-stat strategy has several disadvantages such as slow response to the feed additive and being medium dependent.

The pH-stat operation has previously been used for some other fermentations, ${ }^{5,20}$ but the specific nature of the substrate (sunflower oil in this case) may influence the reliability of this type of operation. When the HAK01 strain was adapted to sunflower oil, no pH change, observed during all cultivation time. This observation is also reported by Sun (2012). ${ }^{20}$

The production of BS in $\mathrm{pH}$-stat mode was measured as $5.9 \mathrm{~g}$ $\mathrm{L}^{-1}$ after $96 \mathrm{~h}$. This is $0.7 \mathrm{~g}$ lower than what obtained in optimized batch operation. So, the overall observation of this part of the experiment, proved that $\mathrm{pH}$-stat mode could not be a suitable choice for BS production by $A$. thermoaerophilus using sunflower oil as a carbon source.

3.4.3. Constant rate feeding. In this part of experiments, two constant rate feeding strategies were evaluated. The first experiment was designed based on oil consumption profile Fig. 2B. As observed in this figure, the oil is consumed in a linear manner during cultivation time according to this equation: $y=19.1-0.103 x$. Where $y$ is the amount of oil $\left(\mathrm{g} \mathrm{L}^{-1}\right)$ in the broth and $x$ is the time (h). The oil consumption rate would be about $0.1 \mathrm{~g}$ per L (broth) per hour or in another word $2.4 \mathrm{~g}$ per L (broth) per day. Since the preliminary experiments showed the importance of yeast extract in the bacterial growth and BS production, in the second experiment, besides constant rate feeding of $2.4 \mathrm{~g}$ per $\mathrm{L}$ per day sunflower oil, yeast extract was also fed to the fermenter at the constant rate of $1.25 \mathrm{~g}$ per $\mathrm{L}$ per day. The rate of feeding was selected with the assumption that all initial yeast extract in the culture is consumed each day.

Fig. 3(A) and (B) show the experimental values of cell growth in term of optical density as well as BS production. In both cases, the feeding of sunflower oil or yeast extract were initiated after $24 \mathrm{~h}$ of a batch phase. As can be observed in this figure, both cell growth and BS production improved compared to batch mode Fig. 1. For the first experiment, the optical density was doubled compared to batch mode and reached to about 6 . For the second experiment, OD reached to about 8. This observation shows the significant effect of feeding on the bacterial growth. The BS production for these two above mentioned experiments obtained as 7.1 and $7.8 \mathrm{~g} \mathrm{~L} \mathrm{~L}^{-1}$, respectively.

Constant rate feeding of essential nutrients is relatively easy to implement and has been widely used in producing biomaterials. However, very few studies have been performed on the investigation of BS production using this method. Kim et al. (2009) investigated the production of sophorolipid with constant rate feeding of glucose using Candida bombicola ATCC $22214 .^{21}$ In this work, rapeseed oil was used as a secondary substrate. As a result, crude sophorolipid up to $365 \mathrm{~g} \mathrm{~L}^{-1}$ in 8 days has been obtained which showed 1.8 times improvement compared to original experiments. However, Chen et al. (2007) reported different results. ${ }^{22}$ They observed that rhamnolipid biosurfactant production has been decreased from $5.3 \mathrm{~g} \mathrm{~L}^{-1}$ in batch mode to $3.05 \mathrm{~g} \mathrm{~L}^{-1}$ in constant rate feeding strategy. In

Table 2 Effect of lack of each essential component in the culture on the growth and BS production

\begin{tabular}{|c|c|}
\hline Lack of the component & Growth \\
\hline $\mathrm{MgSO}_{4}$ & The lack of significant growth \\
\hline Oil & $\begin{array}{l}\text { Low growth during the early hours due to the presence of yeast extract } \\
\text { and then stop growing to day } 4\end{array}$ \\
\hline Yeast extract & $\begin{array}{l}\text { Up to } 6 \text { hours there is no growth. After the matching bacteria with environment, } \\
\text { growth begins on oil }\end{array}$ \\
\hline $\mathrm{NaNO}_{3}$ & Slowdown in growth rate \\
\hline $\mathrm{KH}_{2} \mathrm{PO}_{4}$ & Slowdown in growth rate \\
\hline
\end{tabular}



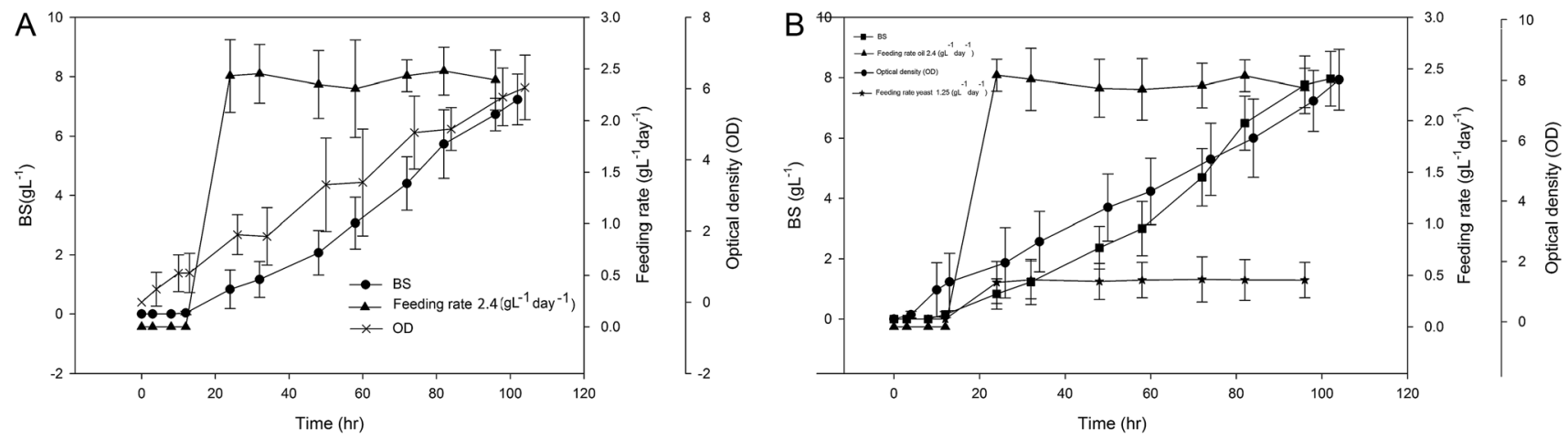

Fig. 3 Constant feeding rate strategy (A) $2.4 \mathrm{~g}$ per $\mathrm{L}$ per day oil, (B) $2.4 \mathrm{~g}$ per $\mathrm{L}$ per day oil and $1.25 \mathrm{~g}$ per $\mathrm{L}$ per day yeast extract.

our case, the BS production improved for about 10 and $17 \%$ for these two experiments. However, it should be noted that, in the constant rate feeding experiments, no DO control was performed.

3.4.4. DO-stat. The result of DO-stat strategy has been presented in Fig. 4. In this setup, nutrient feeding was automated through feedback control of changing values of DO during the fermentation. This feedback parameter was chosen based on the principle that when the carbon/nitrogen sources or other essential components become limiting in a culture, cellular respiratory activity slows down, resulting in a rapid increase in $\mathrm{DO}^{23}$ In this condition and when the dissolved oxygen $(\mathrm{DO})$ rose above the upper limit $(\mathrm{DO}=25 \%)$ as a result of substrate depletion, the nutrient pump was activated. The nutrient pump was deactivated when DO dropped below-set points. However, when the DO dropped drastically below $15 \%$, the agitation speed or aeration was increased. The intense decrease in DO concentration is due to high growth of cells, so there is more need for oxygen in the fermenter.

As can be seen in Fig. 4, in early hours of cultivation an intense fluctuation in the amounts of DO is observed. In the first hours, the amount of DO was controlled around $25 \%$ by both yeast extract feeding and an increase of agitation speed (rpm). The fermentation was relatively well controlled as evidenced by the profiles of cell growth and dissolved oxygen. It should be noted that when the bacterium adapted to carbon source (oil), the fluctuations in the amount of DO was low. DO drop in some defined times so it was adjusted by agitation speed and aeration rate as indicated in Fig. 4 . The BS production in the DO-stat strategy was $8.8 \mathrm{~g} \mathrm{~L}^{-1}$ after $96 \mathrm{~h}$ cultivation time. This was a great result that showed $80 \%$ improvement compared to ordinary batch experiment. On the other hand, the cell growth was drastically increased in this kind of strategy. As seen, the optical density reached to about 33, which is about 10 times greater than batch experiments. This observation shows that controlling the dissolved oxygen in the broth has a great influence on the cell growth and BS production by $A$. thermoaerophilus.

3.4.5. Combined feeding. As stated before, different feeding strategies have been developed including the use of a constant rate feeding, pH-stat, and DO-stat strategies. However, the feeding strategy may be modified during the course of a fed-batch growth to achieve different outcomes.

Since the best results obtained in DO-stat strategy, a combined feeding strategy was designed based on DO-stat method. Therefore, besides keeping the dissolved oxygen in the fermenter at about $25 \%$, feeding of some essential nutrients was applied. Fig. 5 shows the result of this special feeding

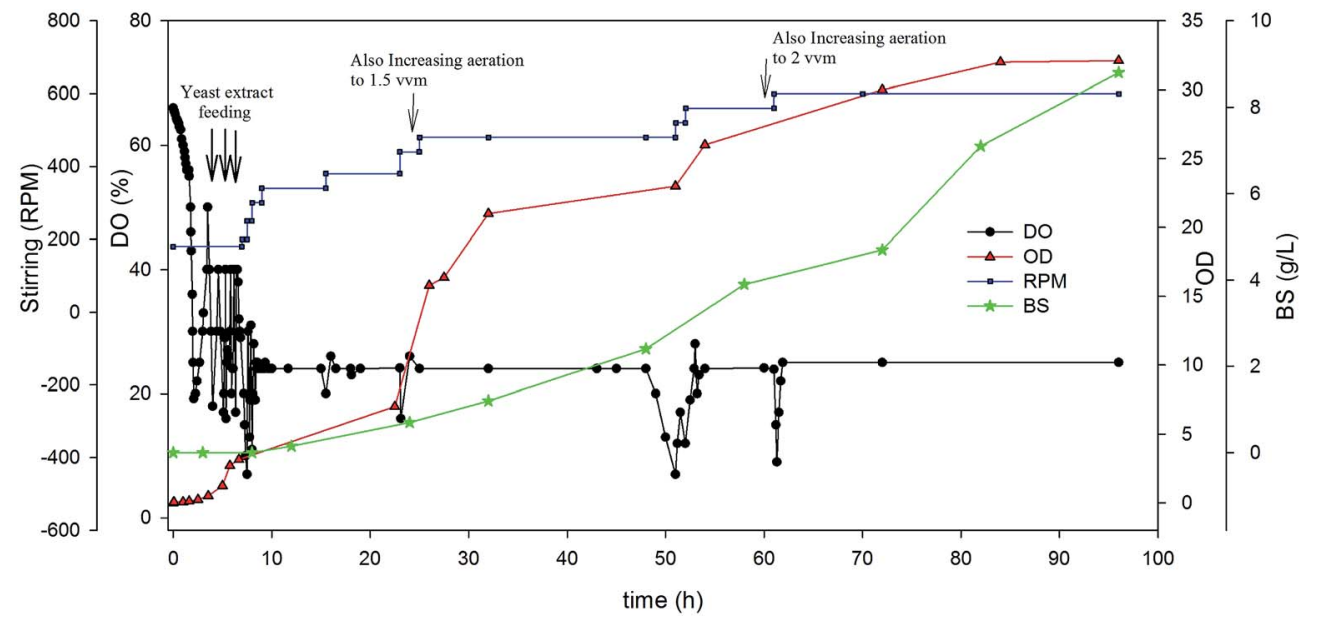

Fig. 4 Variation of parameters over cultivation time in DO-stat strategy. 


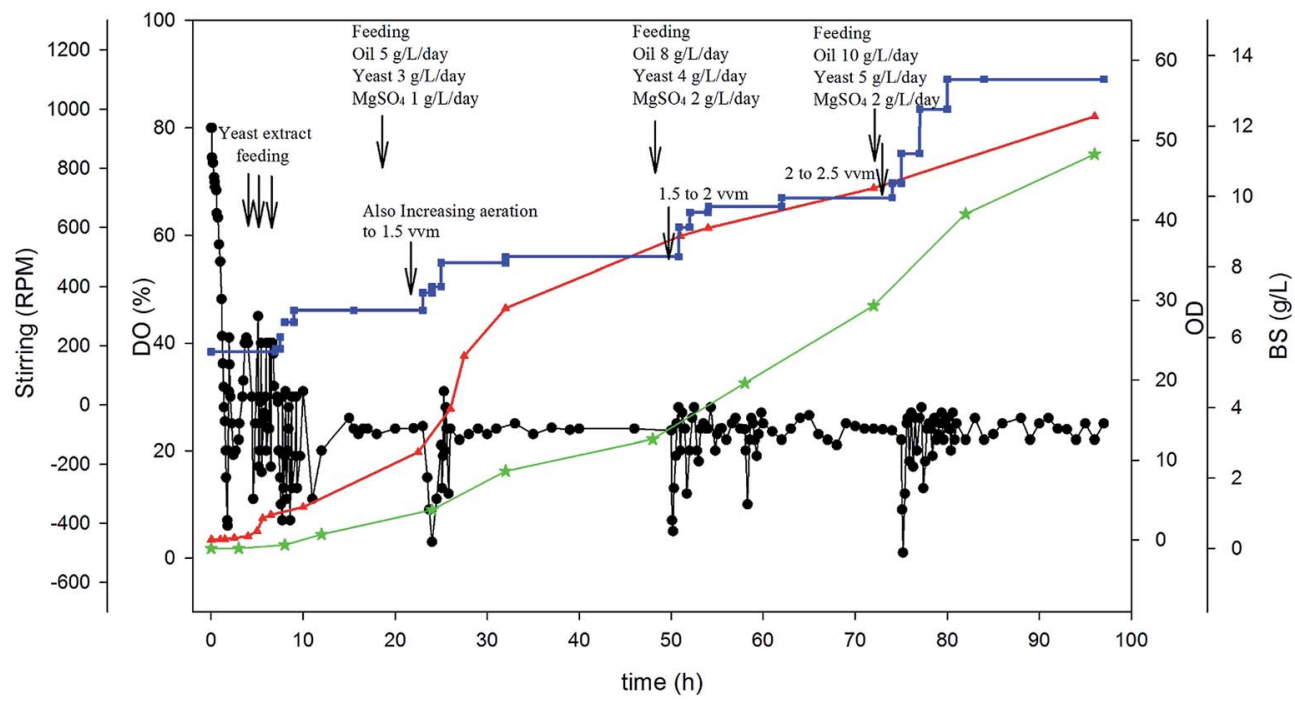

Fig. 5 Variation of parameters over cultivation time in combined feeding rate strategy.

strategy. In this experiment, increasing step-wise feeding of sunflower oil, yeast extract and magnesium sulfate was applied. As observed in Fig. 5, after 24, 48 and $72 \mathrm{~h}$, a specified amount of essential nutrients has been pumped into the fermenter from concentrated nutrient tanks. The fluctuation in DO was controlled by feeding of essential nutrients and changing the stirring and aeration rate. The cultivation started with stirring rate of $180 \mathrm{rpm}$ and an aeration rate of $1 \mathrm{vvm}$ and ended with $1200 \mathrm{rpm}$ and $2.5 \mathrm{vvm}$ due to high cell growth rate. The growth rate was very high compared to the other feeding strategies and reached to an optical density of about 55 after 96 hours. This combined feeding strategy was led to the production of $11.2 \mathrm{~g}$ $\mathrm{L}^{-1}$ BS, which shows 2.28 times increase in BS production compared to batch experiment.

Although combined feeding strategy is more efficient, the study of BS production with this strategy is very scarce. Chen et al. (2007) evaluated pH-stat and constant rate feeding strategies for rhamnolipid biosurfactant production. ${ }^{22}$ Based on the obtained results, they designed a combined feeding that increased BS production from 5.3 to more than $9 \mathrm{~g} \mathrm{~L}^{-1}$.

The summary of the results obtained in this study can be observed in Table 3. Fed-batch fermentation inherits the advantages of achieving high cell density and avoiding substrate or product inhibition. As seen, the production of BS

Table 3 Comparison of BS production in different operational modes

\begin{tabular}{ll}
\hline Operational mode & $\begin{array}{l}\text { BS production } \\
\left(\mathrm{g} \mathrm{L}^{-1}\right) \text { after } 96 \mathrm{~h}\end{array}$ \\
\hline Batch & 4.9 \\
Optimized batch & 6.6 \\
pH-stat & 5.9 \\
Constant feeding rate (oil) & 7.1 \\
Constant feeding rate (oil + yeast) & 7.8 \\
DO-stat & 8.8 \\
Combined feeding & 11.2
\end{tabular}

improved from $4.9 \mathrm{~g} \mathrm{~L}^{-1}$ in batch mode to $11.2 \mathrm{~g} \mathrm{~L}^{-1}$ in combined feeding fed-batch strategy.

\section{Conclusion}

The aim of this study was to minimize substrate inhibition, achieve rapid growth and obtain a high cell density while managing oxygen supply. Different operational modes including, batch, constant rate feeding, $\mathrm{pH}$-stat, DO-stat and combined feeding strategy were evaluated for production of lipopeptide kind of BS. The production of lipopeptide biosurfactant was increased step by step from $4.9 \mathrm{~g} \mathrm{~L}^{-1}$ in batch mode to $11.2 \mathrm{~g} \mathrm{~L}^{-1}$ in combined feeding strategy, which shows $128 \%$ increase in lipopeptide production. $\mathrm{pH}$-stat mode showed weak results and it confirmed that this mode is not applicable to BS production improvement by Aneurinibacillus thermoaerophilus. On the other hand, DO-stat mode showed excellent results in the production of BS. The overall results of the study confirmed the importance of operational mode, oxygen supply and the kind of feeding in lipopeptide biosurfactant production.

\section{Conflicts of interest}

There are no conflicts to declare.

\section{References}

1 H. Hajfarajollah, S. Mehvari, M. Habibian, B. Mokhtarani and K. A. Noghabi, RSC Adv., 2015, 5, 33089-33097.

2 H. Hajfarajollah, P. Eslami, B. Mokhtarani and K. Akbari Noghabi, Biotechnol. Appl. Biochem., 2018, 65, 1-40.

3 S. Anvari, H. Hajfarajollah, B. Mokhtarani and K. A. Noghabi, RSC Adv., 2015, 5, 91836-91845. 
4 H. C. Vogel and C. L. Todaro, Fermentation and Biochemical Engineering Handbook, Noyes publication, New jersey, 2nd edn, 1997.

5 J. Lee, S. Y. Lee, S. Park and A. P. J. Middelberg, Biotechnol. Adv., 1999, 17, 29-48.

6 N. He, T. Wu, J. Jiang, X. Long, B. Shao and Q. Meng, Colloids Surf., B, 2017, 157, 317-324.

7 K. Shimizu, Adv. Biochem. Eng./Biotechnol., 2005, 50, 65-84.

8 M. N. I. Salehmin, M. S. M. Annuar and Y. Chisti, Bioprocess Biosyst. Eng., 2013, 36, 1527-1543.

9 L. Zhu, X. Yang, C. Xue, Y. Chen, L. Qu and W. Lu, Bioresour. Technol., 2012, 117, 208-213.

10 J. S. Guez, S. Chenikher, J. P. Cassar and P. Jacques, J. Biotechnol., 2007, 131, 67-75.

11 S. Bazsefidpar, B. Mokhtarani, R. Panahi and H. Hajfarajollah, Biodegradation, 2019, 30, 59-69.

12 H. Sharafi, M. Abdoli, H. Hajfarajollah, N. Samie, L. Alidoust, H. Abbasi, J. Fooladi, H. S. Zahiri and K. A. Noghabi, Appl. Biochem. Biotechnol., 2014, 173, 12361249.
13 S. Chakravorty, D. Helb, M. Burday, N. Connell and D. Alland, J. Microbiol. Methods, 2007, 69, 330-339.

14 S. F. Altschup, W. Gish, T. Pennsylvania and U. Park, J. Mol. Biol., 1990, 215, 403-410.

15 R. Khademolhosseini, A. Jafari and M. Mousavi, RSC Adv., 2019, 9, 7932-7947.

16 M. N. I. Salehmin, M. S. M. Annuar and Y. Chisti, Biochem. Eng. J., 2014, 85, 8-14.

17 H. Hajfarajollah, B. Mokhtarani and K. A. Noghabi, Appl. Biochem. Biotechnol., 2014, 174, 2725-2740.

18 H. Hajfarajollah, B. Mokhtarani, H. Mortaheb and A. Afaghi, J. Food Sci. Technol., 2015, 52, 3273-3282.

19 S. Nagal and P. C. Jain, Brazilian J. Microbiol., 2010, 41, 196200.

20 J. Sun, Methods Mol. Biol., 2012, 834, 233-244.

21 Y. B. Kim, H. S. Yun and E. K. Kim, Bioresour. Technol., 2009, 100, 6028-6032.

22 S. Y. Chen, Y. H. Wei and J. S. Chang, Appl. Microbiol. Biotechnol., 2007, 76, 67-74.

23 W. Chen, C. Graham and R. B. Ciccarelli, J. Ind. Microbiol. Biotechnol., 1997, 18, 43-48. 